\title{
ON A STATIONARY APPROACH TO SCATTERING PROBLEM ${ }^{1}$
}

BY S. T. KURODA

Communicated by P. Lax, April 1, 1964

1. Let $H_{p}, p=1,2$, be self-adjoint operators in a Hilbert space $\mathfrak{S}$ satisfying the condition

$$
\left(H_{1}-z\right)^{-1}-\left(H_{0}-z\right)^{-1} \in T(\mathfrak{S}), \quad z \in \rho\left(H_{0}\right) \cap \rho\left(H_{1}\right) .
$$

Here, $T(\mathfrak{S})$ denotes the trace class of completely continuous operators in $\mathfrak{S}$ and $\rho\left(H_{p}\right)$ the resolvent set of $H_{p}$. The perturbation theory of absolutely continuous (abbr. a.c.) parts of $H_{p}$ as well as the theory of wave and scattering operators has recently been studied independently by de Branges [2], Birman and Kreĭn [1], and Kato [3]. In [1] and [3] the problem was considered from the viewpoint of the scattering theory. In particular, the wave operators $W_{ \pm}$were proved to exist and hence to be partially isometric operators which give the unitary equivalence of a.c. parts of $H_{0}$ and $H_{1}$. In [2], on the contrary, similar partially isometric operators $\hat{W}_{ \pm}$were constructed somewhat explicitly and without referring to the limit of wave operator type. The purpose of the present note is to study the latter approach from a viewpoint of the scattering theory and to see that the so-called time-independent or stationary approach to the theory of wave and scattering operators can be made possible under the condition (1). In a simpler case, a similar study was made in [4]. Our construction of the operator similar to $\hat{W}_{ \pm}$, i.e. the operator given by the right side of (9), is similar to but slightly different from that given in [2]. In particular, the use of the auxiliary operator $I$ in [2] is avoided. Furthermore, the construction of the operators $\pi_{0}$ and $\pi_{1}$ in 3 might be a little more explicit than that of the corresponding operators given in [2].

2. Let (ㄷ be a separable Hilbert space and let $T_{p} \equiv T_{p}(\mathbb{E}) \subset T(\mathbb{E})$ be the set of all non-negative operators in $T(\mathbb{E})$. The trace norm will generally be denoted by $\tau\left(\right.$ ). Let $\mu$ be a $T_{p}$-valued measure defined for bounded Borel sets of the reals $R^{1}$. Then the set function $\rho$, first defined at each bounded Borel set $e$ as $\rho(e)=\tau(\mu(e))$ and then ex-

${ }^{1}$ The work was partly supported by the National Science Foundation through Purdue University (NSF G-18920). The author wishes to express his thanks to Professor Louis de Branges for valuable discussions and kind hospitality extended to the author while he was visiting Purdue University. 
tended by additivity to the Borel field of $R^{1}$, is a $\sigma$-finite (nonnegative) measure.

The $L^{2}(\mu)$ space over $\mu$ is defined as in [2]. In particular, the set $L_{0}^{2}(\mu)$ of all functions $f(x)$ from $R^{1}$ into ${ }^{5}$ such that $\int_{-\infty}^{\infty}|f(x)|^{2} d \rho(x)$ $<\infty$ forms a dense subset of $L^{2}(\mu)$ (after identification of functions equivalent in a certain sense). The space $L^{2}(\mu)$ can be identified with the direct sum of the $L^{2}$ spaces over the absolutely continuous and singular components of $\mu: L^{2}(\mu)=L^{2}\left(\mu_{a c}\right) \oplus L^{2}\left(\mu_{s}\right)$. The space $L^{2}\left(\mu_{a c}\right)$ is then the a.c. subspace of $L^{2}(\mu)$ with respect to the multiplication operator by $x$ in $L^{2}(\mu)$.

In what follows we assume as in [2] that every $T_{p}$-valued measure $\mu$ satisfies the condition

$$
\int_{-\infty}^{\infty} \frac{1}{1+x^{2}} d \rho(x)<\infty .
$$

For such $\mu$, the $T$-valued function $\phi_{\mu}(z)$ of a complex variable $z$, $\operatorname{Im} z \neq 0$, is defined as

$$
\phi_{\mu}(z)=\frac{1}{\pi} \int_{-\infty}^{\infty} \frac{x z+1}{(x-z)\left(1+x^{2}\right)} d \mu(x) .
$$

(The integral on the right may be regarded as the (improper) integral of a scalar function with respect to a vector-valued measure. Here, we note that the use of the coordinate representation in $\mathbb{5}$ with respect to a complete orthonormal set allows us to make the definition of $L^{2}(\mu)$ space as well as the interpretation of all the integrals appearing in this note by means of the theory of integration of a vector-valued function with respect to a scalar measure.)

Now the following lemma, given in [2] and reformulated below in a slightly different form, will be our starting point.

Lemma. (i) The limits on reals of $\phi_{\mu}(z)$ :

$$
\phi_{\mu}(x \pm i 0)=\lim _{\epsilon \downarrow 0} \phi_{\mu}(x \pm i \epsilon), \quad-\infty<x<\infty,
$$

exist in the Schmidt norm in (5 almost everywhere with respect to the Lebesgue measure.

(ii) Let $\mu$ and $\nu$ be $T_{p}$-valued measures both satisfying the condition such as (2). Let there exist self-adjont operators $\alpha$ and $\beta$ in $(5$ such that

$$
\left\{\alpha+\phi_{\mu}(z)\right\}\left\{\beta+\phi_{\nu}(z)\right\}=\left\{\beta+\phi_{\nu}(z)\right\}\left\{\alpha+\phi_{\mu}(z)\right\}=-1
$$

holds for every nonreal $z$ and put

$$
w(z)=\alpha+\phi_{\mu}(z), \quad w_{ \pm}(x)=w(x \pm i 0) .
$$


Then, the mapping which assigns $w_{ \pm}(x) f(x)$ to each $f(x) \in L_{0}^{2}\left(\mu_{a c}\right)$ is well-defined as an isometric mapping $L_{0}^{2}\left(\mu_{a c}\right)$ onto $L_{0}^{2}\left(\nu_{a c}\right)$ and hence can be extended uniquely to a partially isometric operator $\Omega_{ \pm}$from $L^{2}(\mu)$ into $L^{2}(\nu)$ with the initial set $L^{2}\left(\mu_{a c}\right)$ and the final set $L^{2}\left(\nu_{a c}\right)$. Therefore, if we denote the operators of the multiplication by $x$ in $L^{2}(\mu)$ and $L^{2}(\nu)$ by $A$ and $B$, respectively, then each of $\Omega_{ \pm}$gives the unitary equivalence between the a.c. parts of $A$ and $B$.

(iii) Under the same assumption as in (ii), there exists a uniquely determined unitary operator $T$ from $L^{2}(\mu)$ onto $L^{2}(\nu)$ such that $T$ maps $(x-z)^{-1} c \in L_{0}^{2}(\mu)$ to $w(z)(x-z)^{-1} c \in L_{0}^{2}(\nu)$ for every nonreal $z$ and $c \in \mathbb{C}$.

Now, the following theorem establishes a connection of $\Omega_{ \pm}$with the "asymptotic limit" of wave operator type.

Theorem 1. Let $\mu, \nu, A, B$ and $T$ be as in the lemma. Then, we have

$$
\Omega_{ \pm}=\operatorname{s}_{t \rightarrow \pm \infty} \operatorname{limp}(i t B) T \exp (-i t A) P
$$

where $P$ is the orthogonal projection in $L^{2}(\mu)$ onto its subspace $L^{2}\left(\mu_{a c}\right)$.

The proof of Theorem 1 is an adaptation of the arguments given in Kato $[3, \S 5]$ which essentially prove Theorem 1 in the case of dim $\mathbb{S}$ $=1$. In particular, we get a kind of representation of $T$ such as (4.5) of $[3]$.

3. We shall next apply the foregoing consideration to the theory of wave operators. We shall begin with the following theorem which is deduced from Theorem 1 in a routine way.

TheOREM 2. Let $H_{p}, p=0,1$, be self-adjoint operators in a Hilbert space $\mathfrak{S}$ and $P_{p}$ the orthogonal projection onto the a.c. subspace $\mathfrak{M}_{p}$ of $\mathfrak{S}$ with respect to $H_{p}$. Furthermore, let there exist a separable Hilbert space $\sqrt{ } \sqrt{ }, T_{p}(\mathfrak{S})$-valued measure $\mu$ and $\nu$, and unitary operators $\pi_{0}$ and $\pi_{1}$ from $\mathfrak{S}$ onto $L^{2}(\mu)$ and $L^{2}(\nu)$, respectively, such that $(A$ and $B$ are used as in Theorem 1): (i) $H_{0}=\pi_{0}^{-1} A \pi_{0}, H_{1}=\pi_{1}^{-1} B \pi_{1}$; and (ii) $\mu$ and $\nu$ satisfy the relation (3) with certain self-adjoint $\alpha$ and $\beta$. Then, the wave operator

$$
W_{ \pm}=\operatorname{s}_{t \rightarrow \pm \infty} \operatorname{limp}(i t H) \exp \left(-i t H_{0}\right) P_{0}
$$

exists if and only if there exists a unitary operator $U_{ \pm}$in $\mathfrak{S}$ such that: (a) $H_{1} U_{ \pm}=U_{ \pm} H_{1}$; and (b) $\lim _{t \rightarrow \pm \infty}\left(\pi_{1}^{-1} T \pi_{0}-U_{ \pm}\right) \exp \left(-i t H_{0}\right) u=0$ for each $u \in M_{0}$. In this case we have

$$
W_{ \pm}=U_{ \pm}^{-1} \pi_{1}^{-1} \Omega_{ \pm} \pi_{0}
$$

and hence $W_{ \pm} \mathfrak{S}=\mathfrak{M}_{1}$. 
We now assume that $H_{0}$ and $H_{1}$ satisfy the assumption (1) and construct $\mu, \nu$ etc. in such a way that they satisfy (i), (ii), (a) and (b) in Theorem 2.

Let $U_{p}=\left(H_{p}-i\right)\left(H_{p}+i\right)^{-1}$ be the Cayley transform of $H_{p}$. Then, the assumption (1) implies that $K=\left(U_{1}-U_{0}\right) U_{0}^{-1} \in T(\mathfrak{S})$ and it is expressible as

$$
K=\sum_{k=1}^{\infty} a_{k}\left(\cdot, \phi_{k}\right) \phi_{k}
$$

where $\left(\phi_{i}, \phi_{j}\right)=\delta_{i j},\left|1+a_{k}\right|=1,\left|a_{k}\right| \neq 0$, and $\sum\left|a_{k}\right|<\infty$. Furthermore, let $H_{p}=\int x d E_{p}(x)$ be the spectral resolution of $H_{p}$ and let $F_{p}(e)=\int_{e}\left(1+x^{2}\right) d E_{p}(x)$ for each bounded Borel set $e$.

Let now $\mathfrak{C}$ be the closed subspace of $\mathfrak{S}$ spanned by $\left\{\phi_{k}\right\}$ and put

$$
\mu(e)=\xi^{*} F_{0}(e) \xi, \quad \nu(e)=\eta^{*} F_{1}(e) \eta
$$

where $\xi$ and $\eta$ be given by

$$
\xi=\sum_{k=1}^{\infty} \xi_{k}\left(\cdot, \phi_{k}\right) \phi_{k}, \quad \eta=\sum_{k=1}^{\infty} \eta_{k}\left(\cdot, \phi_{k}\right) \phi_{k},
$$

with $\left\{\xi_{k}\right\}$ and $\left\{\eta_{k}\right\}$ being square summable sequences to be determined below. $\xi$ and $\eta$ are considered to be operators from $\mathfrak{S}$ to $\mathfrak{S}$ so that $\mu(e) \in T_{p}(\mathfrak{S})$ and $\nu(e) \in T_{p}(\mathfrak{S})$. We further assume that $\alpha$ and $\beta$ in (3) have the form

$$
\alpha=\sum_{k=1}^{\infty} \alpha_{k}\left(\cdot, \phi_{k}\right) \phi_{k}, \quad \beta=\sum_{k=1}^{\infty} \beta_{k}\left(\cdot, \phi_{k}\right) \phi_{k}
$$

with bounded real sequences $\left\{\alpha_{k}\right\},\left\{\beta_{k}\right\}$ and want to determine these sequences so that the relation (3) is true. The source of a reciprocal relation such as (3) is the following reciprocal relation in the operator form:

$$
\left\{1+K^{\prime}\left(U_{0}-w\right)^{-1}\right\}\left\{1-K^{\prime}\left(U_{1}-w\right)^{-1}\right\}=1, \quad|w| \neq 1,
$$

where we put $K^{\prime}=U_{1}-U_{0}=K U_{0}$. On the other hand, (4) gives that $\alpha+\phi_{\mu}(z)=\alpha+i \pi^{-1} \xi^{*}\left(U_{0}+w\right)\left(U_{0}-w\right)^{-1} \xi$ with $w=(z-i)(z+i)^{-1}$. By using this and the similar relation for $\nu$ to express (3) in terms of $w$ and comparing it with (7), we have the following proposition.

PROPOSITION. If we put $\xi_{k}=\left|a_{k}\right|^{1 / 2} \xi_{k}^{\prime}$ with an arbitrary sequence $\left\{\xi_{k}^{\prime}\right\}$ of complex numbers such that $0<a \leqq\left|\xi_{k}^{\prime}\right| \leqq b<\infty$ for some positive $a$ and $b$, and determine $\left\{\eta_{k}\right\},\left\{\alpha_{k}\right\}$ and $\left\{\beta_{k}\right\}$ successively by the relations 


$$
\begin{aligned}
2 \xi_{k} \bar{\eta}_{k} & =-\pi a_{k}\left(\bar{a}_{k}+1\right)=\pi \bar{a}_{k}, \\
\alpha_{k} & =i \pi^{-1}\left(1+2 / a_{k}\right)\left|\xi_{k}\right|^{2}, \\
\beta_{k} & =i \pi^{-1}\left(1+2 / \bar{a}_{k}\right)\left|\eta_{k}\right|^{2},
\end{aligned}
$$

then $\mu, \nu, \alpha$, and $\beta$ defined by (4), (5), and (6) satisfy the relation (3). Furthermore, it automatically follows that $\left\{\alpha_{k}\right\}$ and $\left\{\beta_{k}\right\}$ are real and bounded and that $\sum\left|\xi_{k}\right|^{2}, \sum\left|\eta_{k}\right|^{2}<\infty$.

We now construct $\pi_{0}$ and $\pi_{1}$. We can assume without loss of generality that the set of all elements of $\mathfrak{S}$ of the form

$$
u=\sum_{k=1}^{n} u_{k}^{(p)}\left(H_{p}\right) \phi_{k}
$$

with $u_{k}^{(p)}$ such that $\int\left|u_{k}^{(p)}(x)\right|^{2} d\left\|E_{p}(x) \phi_{k}\right\|^{2}<\infty$ forms a dense set in $\mathfrak{S}$ for each $p=0,1$. (The closure of the above set is independent of $p$ and on its orthogonal complement we have $H_{0}=H_{1}$.) For such a $u$ with $p=0$ we define

$$
\left(\pi_{0} u\right)(x)=\sum_{k=1}^{n} \xi_{k}^{-1} u_{k}^{(0)}(x)(x+i)^{-1} \phi_{k}
$$

and $\left(\pi_{1} u\right)(x)$ similarly with $\xi$ replaced by $\eta$. Then, $\pi_{0}$ and $\pi_{1}$ can be uniquely extendable to unitary operators from $\mathfrak{S}$ on $L^{2}(\mu)$ and $L^{2}(\nu)$, respectively. Now the very relation (8) which ensured the validity of (3) also implies the relation $T \pi_{0}=i \pi_{1}$. Thus, we have the following theorem.

Theorem 3. With $\mu, \nu, \alpha$, and $\beta$ defined in the Proposition and $\pi_{p}, p=0,1$, constructed as above, the conditions (i), (ii), (a) and (b) in Theorem 2 hold true. Thus, under the assumption (1), $W_{ \pm}\left(H_{1}, H_{0}\right)$ exists and is given by

$$
W_{ \pm}\left(H_{1}, H_{0}\right)=-i \pi_{1}^{-1} \Omega_{ \pm} \pi_{0}
$$

with $\Omega_{ \pm}$constructed as in Lemma 1.

\section{REFERENCES}

1. M. Sh. Birman and M. G. Kreinn, On the theory of wave and scattering operators, Dokl. Akad. Nauk 144 (1962), 475-478.

2. L. de Branges, Perturbation of self-adjoint transformations, Amer. J. Math. 84 (1962), 543-560.

3. T. Kato, Wave operators and unitary equivalence, Tech. Rep., Univ. of Calif., Berkeley, Calif., 1963.

4. S. T. Kuroda, Finite-dimensional perturbation and a representation of scattering operator, Pacific J. Math. 13 (1963), 1305-1318.

University of Tokyo, Tokyo, Japan 